\title{
A Knowledge Capturing and Sharing Framework for Improving the Testing Processes in Global Product Development Using Storytelling and Video Sharing
}

\author{
Joseph Zammit ${ }^{\mathrm{a}}$, James Gao ${ }^{\mathrm{a}^{*}}$, Richard Evans ${ }^{\mathrm{b}}$, Paul Maropoulos ${ }^{\mathrm{c}}$ \\ ${ }^{a}$ University of Greenwich, Faculty of Engineering \& Science, Chatham Maritime, Kent, ME4 4TB, UK \\ ${ }^{b}$ University of Westminster, Westminster Business School, Marylebone Road, London, NW1 5LS, UK \\ ${ }^{c}$ Aston University, Research and Enterprise, Aston Triangle, Birmingham, B4 7ET, UK
}

\begin{abstract}
In today's global marketplace employee knowledge is seen as a crucial asset for organisations, which enables them to gain a sustainable competitive edge over competitors. Much of the knowledge generated during New Product Development (NPD) and testing can be categorised as tacit, developed from employees' personal experiences and perceptions during Product Development (PD) projects; this makes it more difficult to capture and document for future sharing and re-use. The advent of Social Media in the last decade has changed the way many employees and global organisations communicate and interact with one another, providing a medium for knowledge to be transferred across the World Wide Web in a less formal manner. This research explores whether storytelling and video sharing functionality, embedded into a corporate social media site, is capable of facilitating the capture and sharing of employee knowledge during the PD cycle, providing user feedback on the developed knowledge capture and sharing methodology. It also presents a knowledge framework that is directly driven by the knowledge user, providing both knowledge direction and content.
\end{abstract}

Keywords: Collaboration, Knowledge Management, Product Validation and Testing, Social Media, Video Sharing. 


\section{Introduction}

During NPD, employee knowledge is critical for innovation (1) - to remain competitive in today's global marketplace, knowledge is seen as a crucial asset for organisations which enables them to gain a sustainable competitive edge over rivals through the creation of new innovative products (2). Knowledge Management (KM) can be defined as "the ability to harness and build upon an organisation's intellectual capital" (3). Companies need to identify and record what they know and to use this knowledge effectively. The size and dispersion of global organisations make it especially difficult to locate existing knowledge and deliver it to where it is needed (4).

The creation of new methods of capturing and sharing knowledge amongst PD teams, both local and dispersed, assists companies to capitalise on pre-existing valuable resources; being able to quickly browse and acquire knowledge or to identify knowledge experts within a business can provide a distinct competitive advantage.

The aim of this paper is to explore the use of social media for storytelling and video sharing to facilitate the capture and sharing of employee knowledge during the PD lifecycle. This research is being conducted in collaboration with a globally-dispersed industrial partner operating in the manufacturing sector; this provides insight into current industrial practices relating to the use of social media for $\mathrm{KM}$ in the global manufacturing sector. The paper is organized as follows: Section 2 introduces background information relating to knowledge management, social media and video sharing; Section 3 provides the requirements and a brief overview of the developed knowledge framework; Section 4 describes the approach adopted to implement the developed knowledge framework; Section 5 gives an explanation of the validation of the developed framework followed by 
the results of the validation in section 6; finally, section 7 presents the research conclusions.

\section{Background}

Knowledge can be classified as either Explicit or Tacit. Explicit knowledge can be expressed in formal methods or natural languages and can be easily shared and exchanged. In real-life product development, testing and manufacturing operations, capturing, sharing and making use of on-line explicit knowledge including data and information is very important. For example, in their highly creative experiment, Li et al reported a flexible fixturing and autonomous machining system which responses to the deformation caused by stress release during the machining of large scale aerospace parts $(5-7)$.

Tacit knowledge, on the other hand, cannot normally be easily expressed due to its content, which is constructed from personal skills, experiences and understanding; this makes it difficult to share and exchange by formal and systematic methods (8). Much of the knowledge generated during NPD and testing can be considered tacit, which is connected to problem solving and is dependent on interactions between colleagues within PD teams (9). This type of knowledge is highly abstract and closely related to 'knowhow' (10); thus, one may acquire it in one context and apply and stimulate this knowledge in another $(11,12)$.

Nonaka and Takeuchi (13) argued that tacit knowledge is difficult to capture and share due to one's personal understanding of the subject matter. They stated that only tacit knowledge which can eventually be transformed into explicit knowledge may be successfully shared with others. Hislop (14) suggested that tacit knowledge can be shared 
through "direct communication amongst individuals" and provided three examples of how this may be achieved: 1) stories, 2) observing others and 3) learning by doing within a Community. However, in today's marketplace, accelerated PD timelines to deliver new products in the shortest possible time are critical for success. This generally means that experienced staff have limited opportunities and time to share their own knowledge with younger and less experienced staff (15).

There have been several attempts by researchers to develop new methods to capture and share tacit knowledge. Numerous universities have tried and tested web-based solutions, including eLearning, forums, blogs and video sharing as tools to create a student-centric learning environment, where students themselves create the critical and cognitive skills that higher education aims to develop $(16,17)$. All of these technologies have been used extensively in academic settings with peers using eLearning and social media technologies to capture and/or prepare knowledge content (18). This research uses these same principles to capture and share knowledge; the key difference, however, is that industrial experts are employed to capture and develop the knowledge content. It may be argued that an industrial expert might not have the same level of skills as an academic, but industrial experts are already transferring knowledge on a daily basis to their peers using traditional, direct communication; therefore, they are already transferring knowledge effectively in an informal manner.

Accordingly, it is the researchers' opinion that these industrial experts are the ideal people to capture knowledge, as 1) they are the experts in their fields and 2) if user friendly tools are developed, the required knowledge can be easily captured. 


\subsection{Social Media, Storytelling and Video Sharing tools for Learning and Knowledge}

Transfer

Web 2.0 and social media tools are widely employed today in our daily lives, providing opportunities for people to communicate, learn together and share experiences (19), with software applications such as Facebook, YouTube and Twitter being readily available. Macaskill and Owen (20) defined Web 2.0 as a web-based platform which allows users to gain access, contribute, describe, harvest, tag, annotate and bookmark Web-mediated contents in various formats; anyone with minimal ICT skills can contribute and share their information (21). In recent years, Web 2.0 tools have been used by industry to facilitate intra and inter-firm activities, such as communities of practice/interest, collaborative product development, and open-innovation, inviting external stakeholders to contribute to the NPD process (22).

Reamy (23) suggested that storytelling is the best way to transfer tacit knowledge, conveying information and context that people can understand. According to LeBlanc and Hogg (24), stories make information more meaningful, making tacit knowledge more explicit into learnable chunks. Martin-Niemi and Greatbanks (25) also proposed using storytelling embedded into Web 2.0 tools, providing individualised user experiences.

An ideal medium to capture and share storytelling is video sharing. Balcikanli (26) concluded that a video sharing website like YouTube, is an effective learning tool due to its ease of use and connectivity which not only teaches, but also demonstrate the context the material can be applied to.

\section{Industrial Requirements and Created Knowledge Framework}


An extensive industrial investigation was carried out within a global OEM company with design and manufacturing plants in the UK and around the world. The main outcomes of this investigation highlighted the importance of managing in-house knowledge, allowing employees to capture and share their knowledge more cost effectively within the company (27-29). Resulting from the findings of the research was the developed knowledge framework, shown in Figure 1.

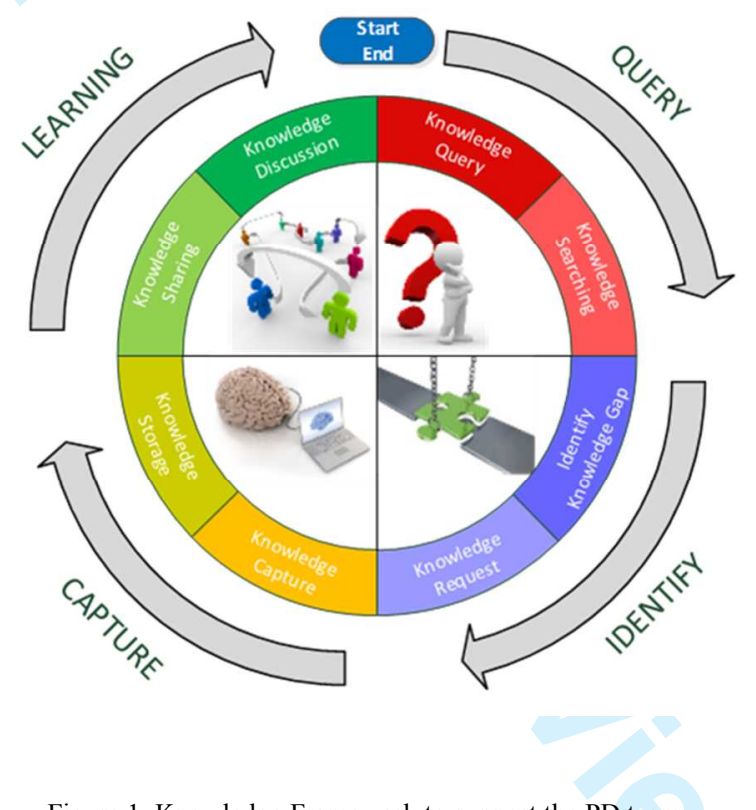

Figure 1. Knowledge Framework to support the PD team.

The knowledge framework represents an iterative knowledge cycle, which not only enables knowledge to be captured and shared within organisations, but also builds upon the knowledge already available; it provides for framework autonomy which informs the knowledge direction, dependent upon end user interests and knowledge needs (30). The framework is made up of four main quadrants: Query, Identify, Capture and Learning, which allow knowledge users to Query the readily available knowledge; if this is not found, a knowledge gap is Identified, activating the knowledge Capturing task by a 
knowledge expert and, finally, the Learning process from the captured knowledge (27). The media requirements for effective knowledge transfer need to be in a format that is easy to use, with the ability to capture complex knowledge content; quick to create and absorb; and allow for different technical levels of competence to understand and use with minimal training (27).

\subsection{Query: Knowledge Search Process in the Knowledge Framework}

Collecting and storing knowledge in electronic format offers resource and cost benefits to organisations. However, it would be pointless if a company is only able to store captured knowledge and not be able to retrieve it in the shortest time possible, on demand and when it is needed. This Section describes the process that knowledge users must follow in order to 1) search and find the knowledge they are seeking, 2) search and find the knowledge and expand it by discussing and questioning the knowledge content, and (3) search and identify a knowledge gap within the knowledge base.

The process starts with the knowledge user having a specific knowledge question, from which they can perform a search. The proposed repository will have five search functions, including: general search, keyword search, look-up of knowledge categories, look-up of specific knowledge contributors and general system browsing. If the user cannot locate the knowledge they are seeking, they will need to identify the knowledge gap and submit a request through the system for a new knowledge contribution. On the other hand, if the knowledge is available in the database, they can absorb and utilise the knowledge content. If the content is sufficient, the search stops here. However, if the knowledge is insufficient, the user has two options. They can either start a discussion with the knowledge contributors or identify a new knowledge gap and submit a new 
knowledge request through the system. The knowledge discussions with the originator can be in the form of either a question to challenge the knowledge or a comment to discuss the knowledge. In both cases, these discussions create direct interactions between the users and the knowledge providers.

\subsection{Identify: Knowledge Gap Request Process in the Knowledge Framework}

A critical component to guarantee the continuation and repetition of the proposed knowledge cycle is the knowledge request process. The aim of this process is to create a formal structure that allows users or administrators to identify and highlight potential knowledge gaps to be addressed by the system. While it was previously stated that the framework should be driven by users, it is recognised that the administrator should also have the ability to identify knowledge gaps and, at the same time, be able to provide the initial stimuli to allow the knowledge cycle to be started, thereby inviting initial contributions for the knowledge database. The process flows are described in more detail below for both user and administrator knowledge requests.

A knowledge request is submitted when a user identifies a gap in the knowledge database. This allows the user to obtain knowledge relating to a specific subject matter. Alternatively, the user can identify a process that, if captured and documented, would raise awareness, be of benefit to colleagues and stakeholders or simply point out improvements to the process.

An additional reason for a knowledge request may involve highlighting product design issues or facility improvements. Sometimes, it is easier to demonstrate an issue rather than to document it in an e-mail or report. The impact of showing a problem is often 
greater than when explaining it in words $(31,32)$, the critical element of this being the changing of written dialogue into visible tangible actions.

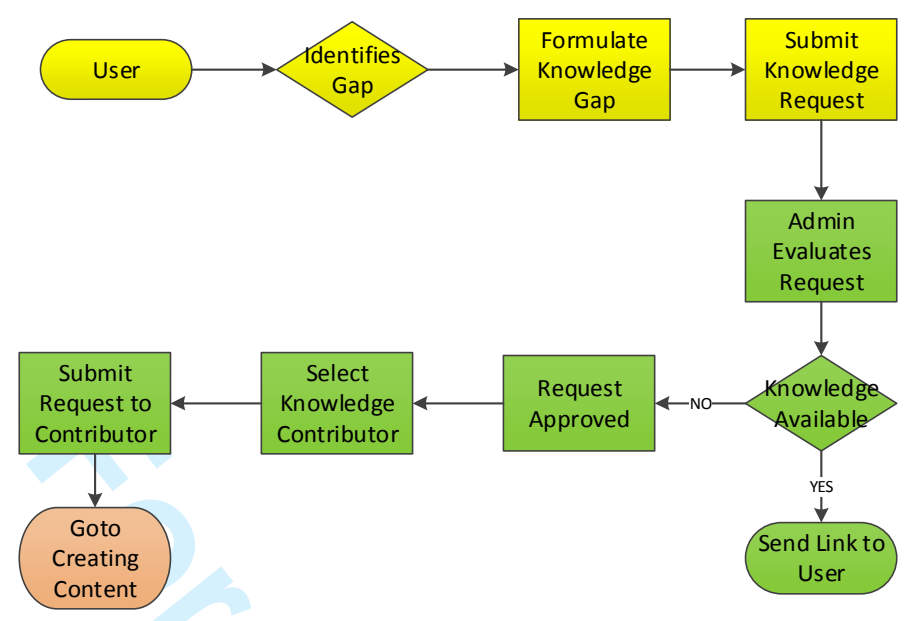

Figure 2. Knowledge user - Knowledge request process flow

The process, illustrated in Figure 2, can generally be used for all previously mentioned knowledge request process. The start of the process is when a user identifies a knowledge gap, from which they specify, in a formal structure, the reason for and benefits of the identified gap. Once this information is uploaded to the system, a user's request can be submitted. Once the request is submitted, the responsibility for completing the request is assigned to the administrator who evaluates the request and checks whether the knowledge already exists in the repository. If it does exist, the administrator provides a link to the knowledge required. On the other hand, if the knowledge is not available and the request is of benefit to the company, the administrator will approve the request, select the most appropriate person to fulfil it and invite the knowledge contributor to create and submit the content.

Initially, the selection of the knowledge contributor is carried out on the basis of the administrator's experience and knowledge of employee expertise. Once the knowledge 
sharing platform is populated with content, experts and active contributors will be identified and categorised by topic and, therefore, will provide an active selection pool from which to choose. A rating scale is available for users to rate the contributions in the system both for knowledge content and knowledge discussion content. This will provide both a quality check, instigated by the users, and a rating of content originators.

\subsection{Capture: Knowledge Capture Process in the Knowledge Framework}

While organisational competitiveness is rooted in the mobility of knowledge that is realised through knowledge sharing and transfer (33), knowledge capture is the critical component required in order to achieve this. The medium selected for knowledge capture in this instance is a combination of video sharing and storytelling. Reamy (23) suggested that storytelling is arguably the best way to transfer tacit knowledge, in that you are able to convey information and context in a form that is easily understood by most. According to LeBlanc and Hogg (24), stories make information meaningful, while tacit knowledge is more explicit and allows information to be organized into learnable chunks.

The proposed process to capture the requested knowledge is illustrated in Figure 3. The starting point of the process begins when a knowledge contributor receives a knowledge request from the administrator, from which information the contributor needs to evaluate the specific needs of the request. If the contributor possesses the required knowledge and skills to deliver the requested contribution, they move on to the next step of planning the knowledge capture. On the other hand, if they do not possess the knowledge requested, they must identify it and acquire this knowledge through available sources, including books, internet searches, equipment manuals and company procedures, and then proceed to the planning stage. 
Once a knowledge contribution plan is created, the user is advised to conduct a relevant literature search to confirm that the knowledge available is up-to-date and relevant, before creating the knowledge contribution plan and submitting it to the administrator. This provides an immediate quality check and avoids wasted time in creating knowledge contributions which are inconsistent with the specified requirements.

Once the knowledge contribution plan has been reviewed and approved by the administrator, the contributor can start collecting the information in any format required to start creating the knowledge story. Once all information is collected, it will be compiled into a single video file with additional voice over to explain the knowledge being shown and provide an alternative visual for learning. This compiled knowledge contribution is then submitted for a second round of approval as a means of quality assurance which, once approved, will be uploaded on to the knowledge sharing platform within the organisation.

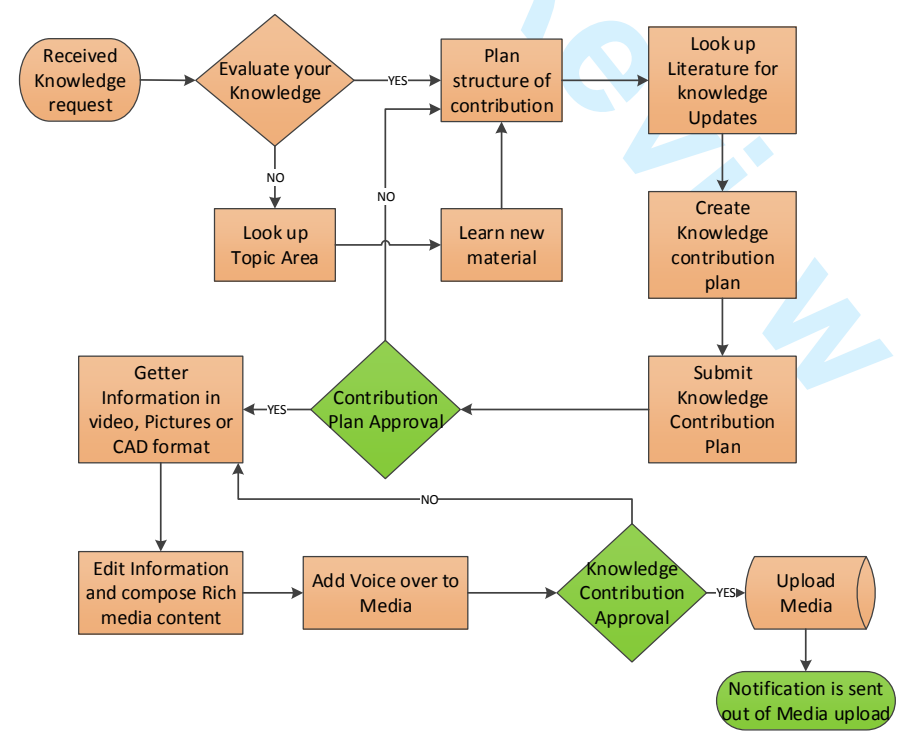

Figure 3. Knowledge contributor - Knowledge capture process flow

\section{Knowledge Capture Development}


The most labour-intensive element of the proposed framework is the knowledge capture process which requires the use of a video capturing device to capture relevant knowledge situations such as processes or demonstrations. An assumption of our research is that video cameras today are widely available and are often integrated into personal communication equipment, such as smartphones, tablets and digital cameras. Therefore, it was assumed that the majority of people have a basic working knowledge of video cameras and photography equipment. Nevertheless, a basic 'How to use a Camera' training video was created to demonstrate this skill.

Once the raw video images had been captured by the knowledge contributor, the next step in the knowledge capturing system was to compile the raw media into a single coherent video, delivering a story told knowledge message. This was achieved by compiling a knowledge video using video editing software. For this project, Adobe Premier Pro was selected due to its availability within the collaborating organisation. The software allowed for the editing of video footage and the addition of special effects and sound recordings. Training material on 'How to use the Video Editing Software' was created using the developed a knowledge capturing methodology.

In order to produce a coherent knowledge video, it was determined that the knowledge contribution needed some form of structure in order to assist the knowledge contributor to build the media, while also helping the knowledge receiver to absorb the knowledge by creating knowledge contributions with common features, such as ease of knowledge understanding and knowledge structure.

The key elements identified to improve a user's understanding of a knowledge contribution are shown in Figure 4. This consists of 1) the video content with voiceover 
explaining what is being demonstrated, 2) the process flow chart of what is being demonstrated, and 3) the physical layout of the system to help the knowledge receiver comprehend the location dynamics. This layout provided the best knowledge format, as reviewed by the system users, as it allowed them to make links and connections to what they were seeing in the video in relation to the physical location and the order in the process tree. This structure was then created into a template shown in figure 5, which was used by the knowledge contributors for the purpose of the case study.

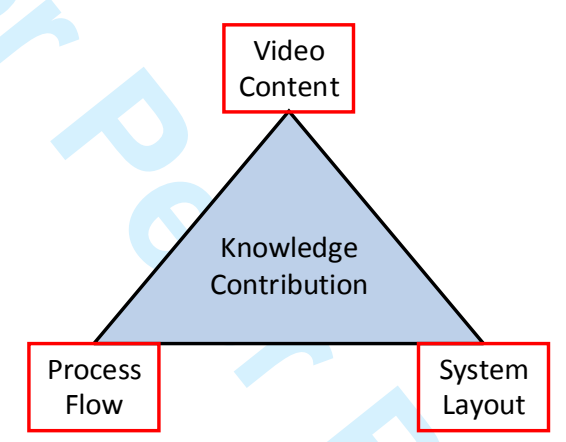

Figure 4. Knowledge contribution main components 


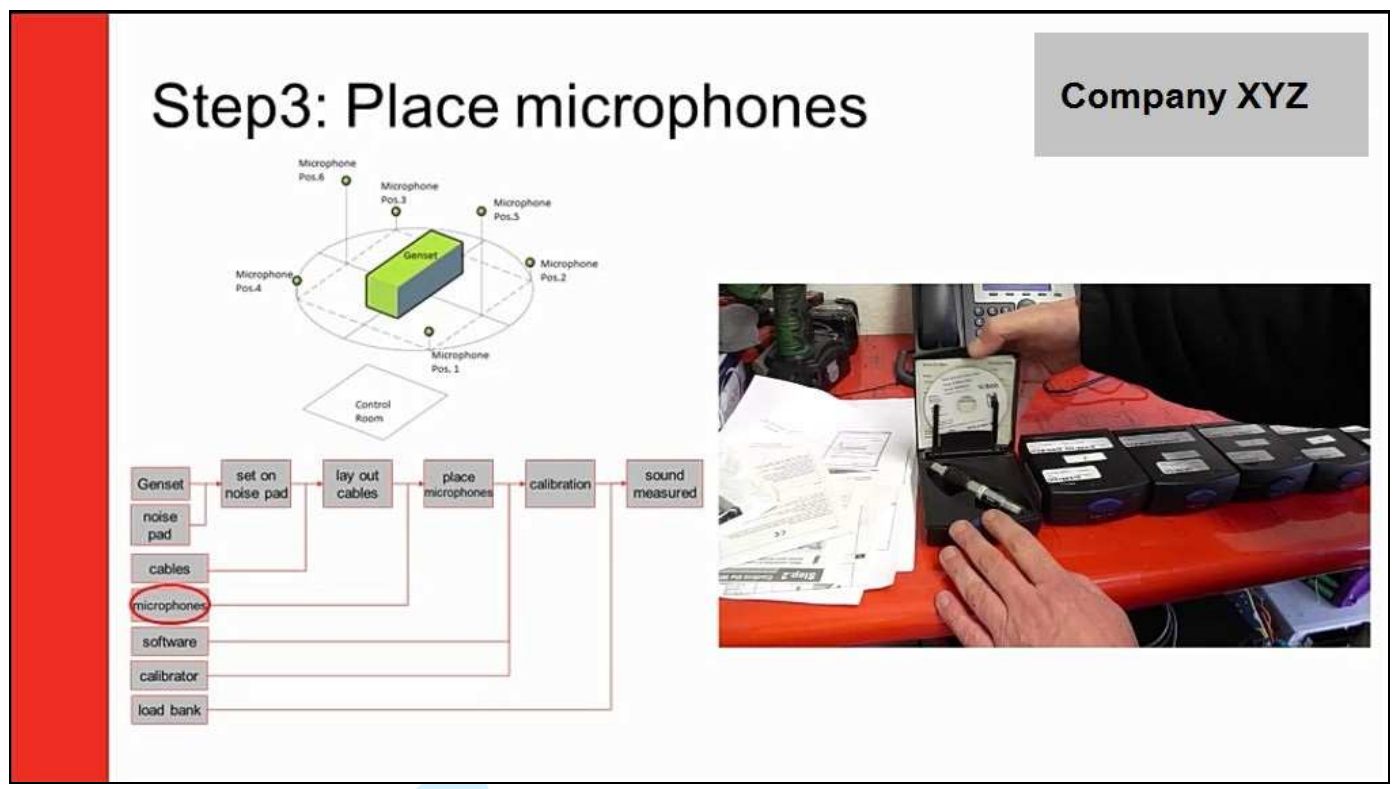

Figure 5. Knowledge contribution template - complete example

Instead of showing the video in full screen, it is shown as split-screen, with the process flow and layout shown to the side. The intention of this was to highlight the location, both in the physical layout and process flow, depending on what stage of the video presentation it was at. This combination allows users to make links and connections to what they are seeing in the video in relation to the physical location and the order in the process tree.

Another important consideration in the design of the system was the duration of the knowledge contribution. During the investigation, users indicated that they preferred to view short knowledge contributions, lasting between $5-10$ minutes each, rather than longer presentations (28). This benefits the attention span of the users; the longer the knowledge contribution, the greater the possibility of users losing interest or even stopping the video halfway. Therefore, a time duration recommendation was put into place and knowledge contributions should be between $5-10$ minutes in length. 


\section{System Validation}

The purpose of the validation exercise was to confirm that the developed framework, methodology and prototype knowledge sharing tool, fulfilled the collaborating company's need to capture, disseminate and transfer knowledge more efficiently and effectively throughout the organisation using rich media and social media tools. This was accomplished by means of a validation study undertaken by a selected number of employees from the collaborating company. The participant selection criteria varied by age groups, ranging from $20+$ to $40+$ and by different levels of skill/educational background, ranging from Technicians, Engineers to Managers, in order to obtain balanced user experience feedback, representative of the workforce within the collaborating organisation.

The validation process consisted of two groups of participants: knowledge contributors and knowledge receivers. The 'knowledge contributor' group consisted of 6 employees, while the 'knowledge receiver' group consisted of 16 employees. The knowledge contributors consisted of 3 engineers and 3 technicians, while the knowledge receivers consisted of 3 managers, 6 engineers and 7 technicians. User feedback was gathered using one-to-one face-to-face interviewing followed by an online survey to gather nondiscussed data. The sample size for the validation process was 22 and, therefore, the results may only be considered indicative, but the feedback obtained is promising and shows strong user acceptance and usability of the system. The validation process consisted of four key stages:

1. The creation of knowledge contributions; 
2. Workshop and survey with knowledge contributors to capture end-user feedback;

3. Workshop and survey with knowledge receivers to capture end-user feedback; and

4. Live workshop with knowledge receivers to assess actual knowledge transfer.

\section{Validation Survey Results and Analysis}

In this section, the results from the four stages of the validation process are presented and analysed in detail from the feedback received from the end-users.

\subsection{Knowledge Contribution}

The first step in creating a knowledge contribution was to identify appropriate knowledge subjects that would provide value and then to identify participants who were considered experts in their subject areas and who could make a valid contribution whilst taking into account the participant selection criteria to reflect the complete workforce from the company. In total, seven topics were selected as possible knowledge contributions, from which six were completed giving an $86 \%$ completion rate.

While the knowledge capture process took longer than anticipated due to the availability of staff and general business priorities, all knowledge contributors found the process innovative. It was noted that while all participants in the study had used to a varying degree social media and digital equipment, those who were more familiar and confident found the task easier than others who were less familiar. This was not due to training material being insufficiently clear or because they lacked the skills required to complete the task, but rather, due to lack of confidence and uncertainty as participants questioned their ability to complete the task. 
From the progress meeting carried out with participants, it was noted that young engineers were more open to sharing their knowledge and not worried about getting it wrong or being criticized on the content that they had created. This could result from their recent university experience where, in general, engineering students are encouraged to work and solve problems together on group projects and where the consequences of mistakes are more forgiving. On the other hand, the older generation were more apprehensive about the knowledge content they created. An additional observation noted during knowledge capture was the issue of language and the effectiveness of knowledge contributions made by non-native English speakers. One participant was a non-native English speaker with a strong accent. The effects of non-native English speaking personnel and strong foreign accents did not appear to make any negative impact on knowledge capture.

\subsection{Workshop Knowledge Contribution User Response}

During this workshop, the six knowledge contributors were asked to rate, against a 5point Likert scale, several items within the knowledge capture process, in order to evaluate the developed methodology. When asked about the difficulty of collecting information and planning for the knowledge contribution, $50 \%$ of participants replied with a difficulty rating of 2 , with the remaining $50 \%$ providing a rating ranging from 4 to 5, describing the process as easy, as shown in Figure 6. When participants were asked to elaborate on why they rated the process so low, the reason given was that they felt that they did not have enough time allocated to work on the knowledge capturing task during their normal working week. This made the task difficult to complete in the specified timeframe. 


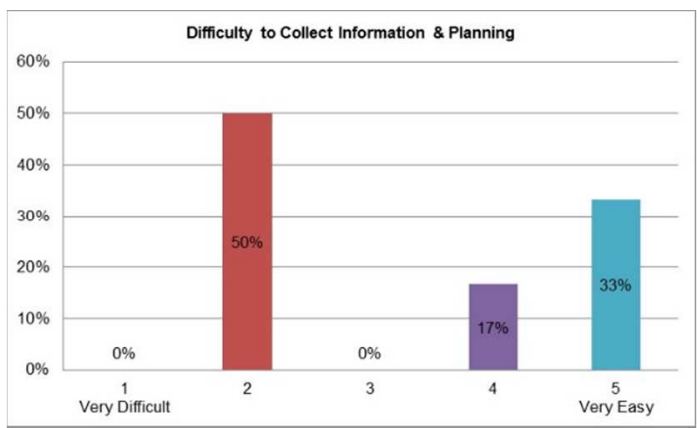

Figure 6. Difficulty to Collect Information \& Planning of Knowledge Contribution Form

The same situation was experienced by participants when preparing for the knowledge contribution, involving the collection of information, writing up and planning for the knowledge capture. $67 \%$ of participants gave a 3 rating, while $33 \%$ of participant gave a 5 rating of very easy, as shown Figure 7 . This again resulted due to not having the right equipment or facilities available when required.

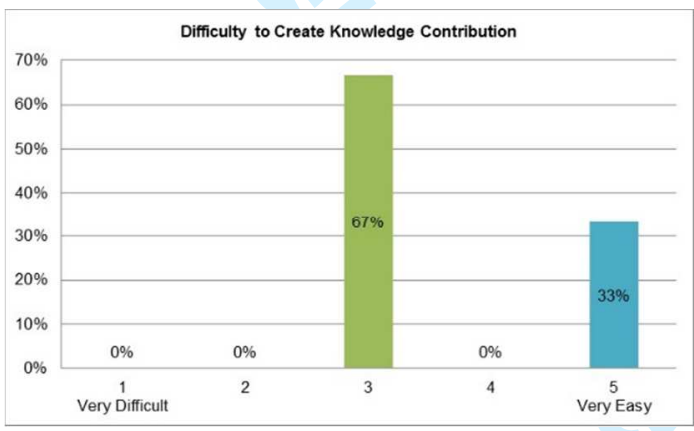

Figure 7. Difficulty to Create Knowledge Contribution

One resultant hypothesis of this project was that the extensive use of social media and smartphones in participants' daily lives means that they have enough skills to use these tools. When participants were asked how difficult they found capturing video content for their knowledge contribution using the supplied video capture equipment, all rated the process as very easy, with $33 \%$ giving a rating of 4 and $67 \%$ giving a rating of 5 , as shown in Figure 8. These results, combined with the quality of video captured from the 
knowledge contributors, provides a good indication that this assumption has some weight and provides some confidence to the statement.

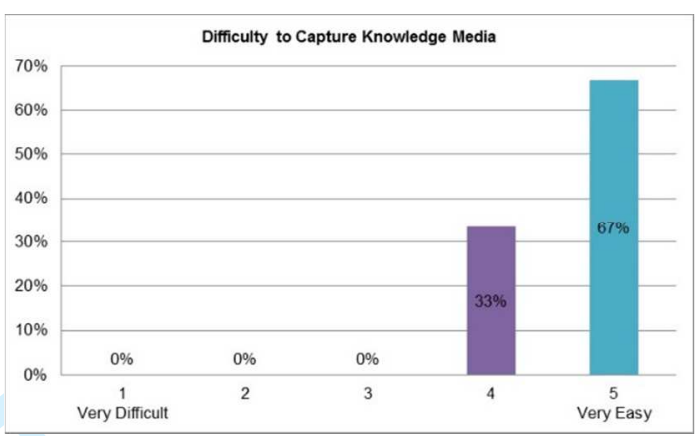

Figure 8. Difficulty to Capture Knowledge Media

With regard to the compilation and editing of the knowledge contribution using the provided video editing software, the participants found that the training material and the usability of the software greatly simplified the process which meant that they did not encounter any problems. The majority of participants gave a rating of 4 and 5 , stating that the process was easy, as can be seen in Figure 9. When asked about the difficulty of the knowledge contribution process, the majority of participants (83\%) gave a rating of 4 .

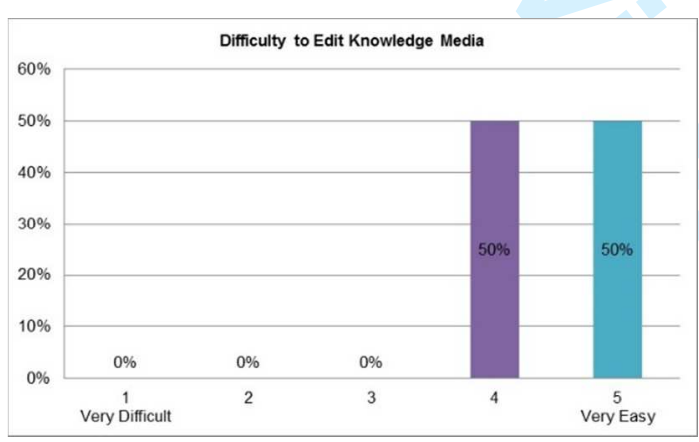

Figure 9. Difficulty to Edit Knowledge Media

In general, the feedback from participants was positive, identifying that knowledge contributions would be a useful tool for knowledge dissemination in the company. The 
main issue that was highlighted during the study was the lack of dedicated time allocation and equipment being readily available as and when required.

\subsection{Workshop Knowledge Receiver User Response}

A second workshop was organised amongst the six knowledge contributors and sixteen knowledge receivers whom were asked to rate, using a 5-point Likert scale, several items within the knowledge sharing process in order to evaluate the developed methodology. After being shown two knowledge contributions created by their colleagues, the knowledge receivers were asked if they found the shared knowledge informative and if they had learned something from watching it. The majority found them very informative, as shown in Figure 10, with 38\% rating the experience as 4 and 63\% rating it as 5 .

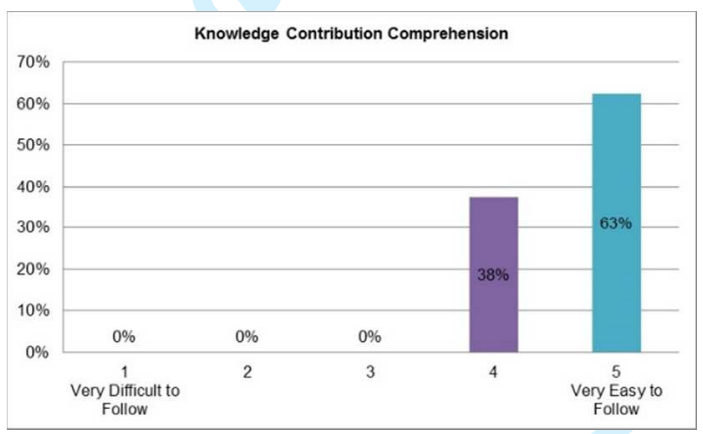

Figure 10. Knowledge Contribution Comprehension

With regard to the quality of knowledge contributions created, they also rated this as of good quality, with $50 \%$ giving a 4 rating and $50 \%$ providing a 5 rating, as shown in Figure 11. Some did comment on the consistency of the sound quality which, in some cases, created a drop in volume during sections; sometimes background noise was audible. This has been attributed to selecting the wrong work space by the knowledge contributors when creating the knowledge contributions. 


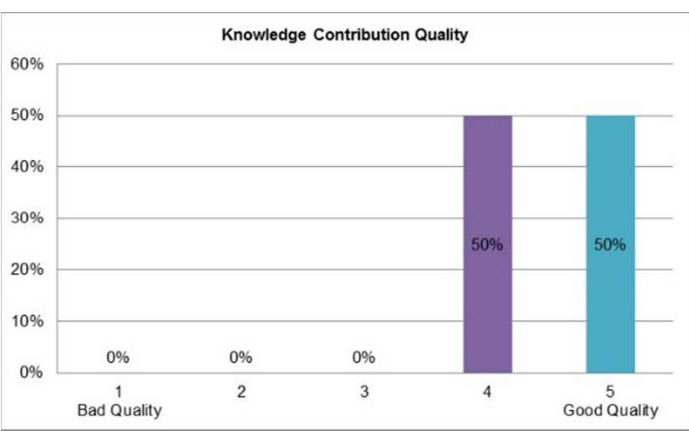

Figure 11. Knowledge Contribution Quality

The length of knowledge contribution videos was also discussed during the workshop. Users expressed differing views, as was found during the initial investigation (28). However, after the users had been shown a knowledge contribution, the majority preferred to view shorter video presentations lasting between 5-10 minutes. The reasons given were that a shorter presentation would increase the likelihood of people using the system and that users would possibly lose interest if longer videos were used.

When participants were asked what improvements they would make to the system, most stated that the knowledge captured was of good quality and that "there was not much to improve". Study participants stated that the system needed greater implementation time in order to mature and expand the number of knowledge contributions captured on the system. Some highlighted the benefit of the user rating system. The fact that the quality and content of any knowledge contribution on the system may be judged and rated made the participants feel included in the decision and quality control process.

When asked whether they saw value in such a system, all agreed that the system would be of benefit to them and the company. When participants were asked if they would contribute towards knowledge discussions using the blog / comments section attached to 
each knowledge contribution, all stated that they would and that they saw great benefit in being able to receive comments from the originator of the knowledge contribution. A positive response was received when asked if participants would use such a system to search for knowledge or contribute towards it, with 38\% giving a rating of 4 and $63 \%$ giving a rating of 5 , showing that employees are 'highly likely' to use such a system.

One critical design concept of the system was to create an environment where knowledge users determine the knowledge direction the system should take in order to reduce the administrative burden and also target the required knowledge to be captured because the knowledge user is asking for it. Therefore, participants were asked to propose a topic that they were interested in learning and would like to see captured. A total of 114 unique topics were identified, which suggests the usefulness and need for this system.

\subsection{Live Workshop to Assess Knowledge Transfer User Response}

The first group that was assessed were the knowledge contributors who needed to follow the developed training material in order to understand what a knowledge contribution entailed and how to create it. The primary success indicator for successful knowledge transfer was the successful completion of a knowledge contribution unaided and which could be considered achieved. The only help participants required was minor hints and tips and encouragement to complete the task. The second indicator related to the quality and the medium that the knowledge was obtained from; the feedback received from the users in the previously discussed workshops, from which positive feedback resulted.

The second group assessment was during the final proof of concept stage, where an observational study was organised with volunteers, who viewed a knowledge contribution created by one of their colleagues and, from which, they were required to 
replicate the task shown without help or guidance from others. This study was carried out in the employees normal working environments in order to simulate a typical working day with phones ringing and people coming in and out of the work space. In all observed studies, the task was replicated correctly even though at different durations. The differences in time taken to replicate the task could be directly attributed to the interruptions experienced and also due to the different levels of knowledge retention of participants. It was noted that some had to repeat portions of the replication, either because they were not paying sufficient attention or the subject matter was more difficult to understand. However, by having the functionality to stop the video, think and continue or stop, rewind and start over again, gave them the independence and autonomy to absorb the knowledge at their own pace and to complete the task successfully.

\subsection{Evaluation of the Developed Knowledge Sharing Framework}

In general, the majority of users responded positively to the use of the developed knowledge repository and the knowledge contributions created using the developed methodology. Positive feedback was also received from participants who captured and created the knowledge contributions. All participants appreciated the benefit to them and the company in contributing to and receiving knowledge from such a KM system.

Considering again the initial requirements when developing a knowledge sharing framework to capture and share explicit to more complex tacit employee knowledge, the validation process has demonstrated that the developed methodology was effective in producing the knowledge contribution initially sought. Analysis has shown that knowledge transfer occurred and end users accepted both the knowledge capture and sharing process. 
User feedback in relation to willingness to search for knowledge in such a system and to contribute towards the system with either knowledge contributions, knowledge direction or through knowledge discussion, suggests that such a tool which is able to capture employee knowledge and provide users with an ability to control the direction and quality of knowledge being stored is of future benefit.

The analysis of the knowledge captured by users reveals that storytelling and video sharing technologies can be used successfully to provide a rich and informative knowledge content medium that enables knowledge sharing and transfer. The validation of actual knowledge transfers from the conducted live workshops 1) during the knowledge capture portion of the project, and 2) during the final proof of concept workshop evaluation process has confirmed this.

The use of social media and Web 2.0 tools to collaborate and discuss complex engineering knowledge meets the requirements of the social aspects of communication and knowledge management; it further informs previously captured knowledge and helps to build and create new knowledge within the knowledge sharing tool. The developed methodology has provided a tool to capture and manage tacit knowledge which may be considered as a contribution towards knowledge in global manufacturing literature. The feedback received demonstrates that the functionality and usability of the developed system can be further enhanced and expanded for use by a larger audience in order to further confirm the system capabilities and functionality.

\section{Conclusion}

The intention of this research was to answer the question of whether social media and video sharing tools were capable of facilitating the capturing and sharing of employee 
knowledge during the NPD testing cycle. Based on this topic, a knowledge capture and sharing framework was developed, which is directly driven by the knowledge user, providing both knowledge direction and content. An OEM company was used as a case study to develop and test this methodology, which can also be applied to other design or manufacturing companies and general enterprise. The novelty of this research lies in the developed methodology to capture and share knowledge, addressing the special nature and application context of integrated PD and testing operations. Similarly, the use of social media, video sharing and storytelling technologies to capture complex engineering knowledge by the knowledge experts themselves, rather than by media professionals whom are paid to develop content, is relatively unique. This should guarantee the organisation more informed knowledge content and a reduction in costs in developing knowledge content.

Another topic explored in this research was the possibility of giving administrative control of knowledge direction and content to the knowledge user as the main driver of the knowledge management system. The main advantage of the developed methodology is that it improves accessibility of knowledge to employees, whilst existing text-based knowledge management systems are considered heavy, laborious, dull and sometimes ignored.

The proposed methodology consists of a knowledge capture and sharing framework, providing the theoretical underpinning of the system, process, procedures and templates to aid knowledge capture. The results of the case study and validation exercise have confirmed that the proposed methodology has the capacity to develop a comprehensive KM system to manage both knowledge and procedures, based on business and user 
requirements. Comments suggest that the proposed methods aid employees in their task of capturing knowledge, while already demonstrating value in having the knowledge electronically stored and readily available for knowledge transfer.

\subsection{Future Work}

This project was conducted in collaboration with an OEM organisation, with the main sponsor championing the project, being the product development testing facility. While an extensive case study has been conducted with different participants at different levels of the company hierarchy, a wider study should be conducted in other areas of the business; this would provide the possibility to continue to verify the flexibility and simplicity of the developed tool and also provide greater awareness across the whole company and provide an easy and accessible portal to cross train the different functions.

The developed methodology has been developed with general use in mind, so that it can be applied across different business units within the enterprise and can be applied to industry in general. This can be considered as a short coming, by not generalizing the validation of the framework across different industries. Further research could be conducted to explore other industrial setups to validate the envisaged usage of the methodology.

The effect of having knowledge contributions created by non-native English speakers was also briefly explored during the knowledge capture phase. As an objective of the tool is that it be used by multiple people spread out in a global enterprise the effectiveness of knowledge contributions created by non-native English speakers should also be further validated to confirm the initial findings. 


\section{References}

1. Nakata C, Benedetto CA. Forward to the Future: The New Knowledge Needed to Advance NPD $\square$ Innovation Theory and Practice. Journal of Product Innovation Management. 2012;29(3):341-3.

2. Hau YS, Kim B, Lee H, Kim Y-G. The effects of individual motivations and social capital on employees' tacit and explicit knowledge sharing intentions. International Journal of Information Management. 2013;33(2):356-66.

3. Drucker P. The New Realities: Transaction Publishers; 2011.

4. Davenport TH, Prusak L. Working Knowledge: How Organizations Manage what They Know: Harvard Business School Press; 2000.

5. Zhou Y, Li Y, Wang W. A feature-based fixture design methodology for the manufacturing of aircraft structural parts. Robotics and Computer-Integrated Manufacturing. 2011;27(6):986-93.

6. Liu C, Li Y, Gao X. Feature-based adaptive numerical control programming method for the environment of changing manufacturing resources. Proceedings of the Institution of Mechanical Engineers, Part B: Journal of Engineering Manufacture. 2015;13(5):237-47.

7. Li Y, Wang W, Li H, Ding Y. Feedback method from inspection to process plan based on feature mapping for aircraft structural parts. Robotics and Computer-Integrated Manufacturing. 2012;28(3):294-302.

8. Venkitachalam K, Busch P. Tacit knowledge: review and possible research directions. Journal of Knowledge Management. 2012;16(2):357-72. 
9. Goffin K, Koners U. Tacit knowledge, lessons learnt, and new product development. Journal of Product Innovation Management. 2011;28(2):300-18.

10. Grant RM. Toward a Knowledge-based Theory of the Firm. Strategic Management Journal. 1996;17:109-22.

11. Burrows B. Common Knowledge: How Companies Thrive On Sharing What They Know: Nancy M. Dixon, Harvard University Press (2000), 188 pp. npq. Long Range Planning. 2001;34(2):270-3.

12. Nonaka IT, R. The Knowledge-creating theory revisited: Knowledge creation as asynthesizing process. Knowledge Management Research and Practice. 2003;1(1):2-10.

13. Nonaka I, Takeuchi H. The Knowledge-creating company. Oxford, UK: OxFord University Press; 1995.

14. Hislop D. Mission impossible? Communicating and sharing knolwedge via information technology. Journal of Information Technology. 2002;17(3):165 - 77.

15. Mueller J. A specific knowledge culture: Cultural antecedents for knowledge sharing between project teams. European Management Journal. 2014;32(2):190-202.

16. Jonassen D, Mayes T, McAleese R. A manifesto for a constructivist approach to uses of technology in higher education. Designing environments for constructive learning: Springer; 1993. p. 231-47.

17. Moron-Garcia S. Using virtual learning environments: lecturers' conceptions of teaching and the move to student-centred learning. International Conference on Computers in Education: IEEE; 2002. p. 1494 - 5.

18. Clark RC, Mayer RE. e-Learning and the Science of Instruction: Proven Guidelines for Consumers and Designers of Multimedia Learning: Wiley; 2011. 
19. Elia G, Margherita A, Taurino C. Enhancing managerial competencies through a wiki-learning space. International Journal of Continuing Engineering Education and Life Long Learning. 2009;19(2-3):166-78.

20. Macaskill W, Owen D, editors. Web 2.0 to go. Proceedings of LIANZA Conference; 2006.

21. Evans RD, Gao JX, Martin N, Simmonds C, editors. Using Web 2.0-based groupware to facilitate collaborative design in engineering education scheme projects. 2014 International Conference on Interactive Collaborative Learning (ICL); 2014 3-6 Dec. 2014.

22. Evans RD, Gao JX, Mahdikhah S, Messaadia M, Baudry D. Crowdsourcing UserContributed Solutions to Aerospace Product Development Issues through MicroBlogging. Electronic Journal of Knowledge Management. 2016;14(2):126-36.

23. Reamy T. Imparting knowledge through storytelling, part 1 of a two-part article. KMWorld Magazine. 2002;11(6).

24. LeBlanc SM, Hogg J. Storytelling in knowledge management: an effective tool for uncovering tacit knowledge. Society for Technical Communication processing, Atlanta. 2006.

25. Martin-Niemi F, Greatbanks R. SME knowledge transfer through social networking: Leveraging storytelling for improved communication. 1st International Conference on Computer-Mediated Social Networking, ICCMSN 2008. Dunedin2009. p. $86-92$. 
26. Balcikanli C, editor Long live, YouTube: L2 stories about YouTube in language learning. Annals of Language and Learning: Proceedings of the 2009 International Online Language Conference (IOLC 2009); 2010: Universal-Publishers.

27. Zammit JP, Gao J, Evans R. A Framework to Capture and Share Knowledge Using Storytelling and Video Sharing in Global Product Development. Product Lifecycle Management in the Era of Internet of Things: 12th IFIP WG 51 International Conference, PLM 2015,. Cham: Springer International Publishing; 2016. p. 259-68.

28. Zammit J, Gao JX, Evans R, Saunders T, editors. Investigating the use of social media in improving knowledge management within a collaborative product development and testing environments. International Conference on Manufacturing Research (ICMR 2015); 20158 - 10 September; University of Bath2015.

29. Zammit J, Gao JX, Shah S, editors. A knowledge sharing framework for improving the testing processes in global product development. International Conference on Manufacturing Research (ICMR 2014); 20149 - 11 September; Solent University2014.

30. Zammit J, Gao JX, Evans R. Capturing and Sharing Product Development Knowledge using Storytelling and Video Sharing. DET 2016 - The 9th International Conference on Digital Enterprise Technology; 29 -31 March 2016; Nanjing, China. Special Issue Procedia CIRP: Procedia CIRP; 2016.

31. Tapp LM, editor A Picture is Worth a Thousand Words. ASSE Professional Development Conference and Exposition; 2014: American Society of Safety Engineers.

32. Yadav A, Phillips MM, Lundeberg MA, Koehler MJ, Hilden K, Dirkin KH. If a picture is worth a thousand words is video worth a million? Differences in affective and 
cognitive processing of video and text cases. Journal of Computing in Higher Education. 2011;23(1):15-37.

33. Cummings JN. Work groups, structural diversity, and knowledge sharing in a global organization. Management science. 2004;50(3):352-64. 


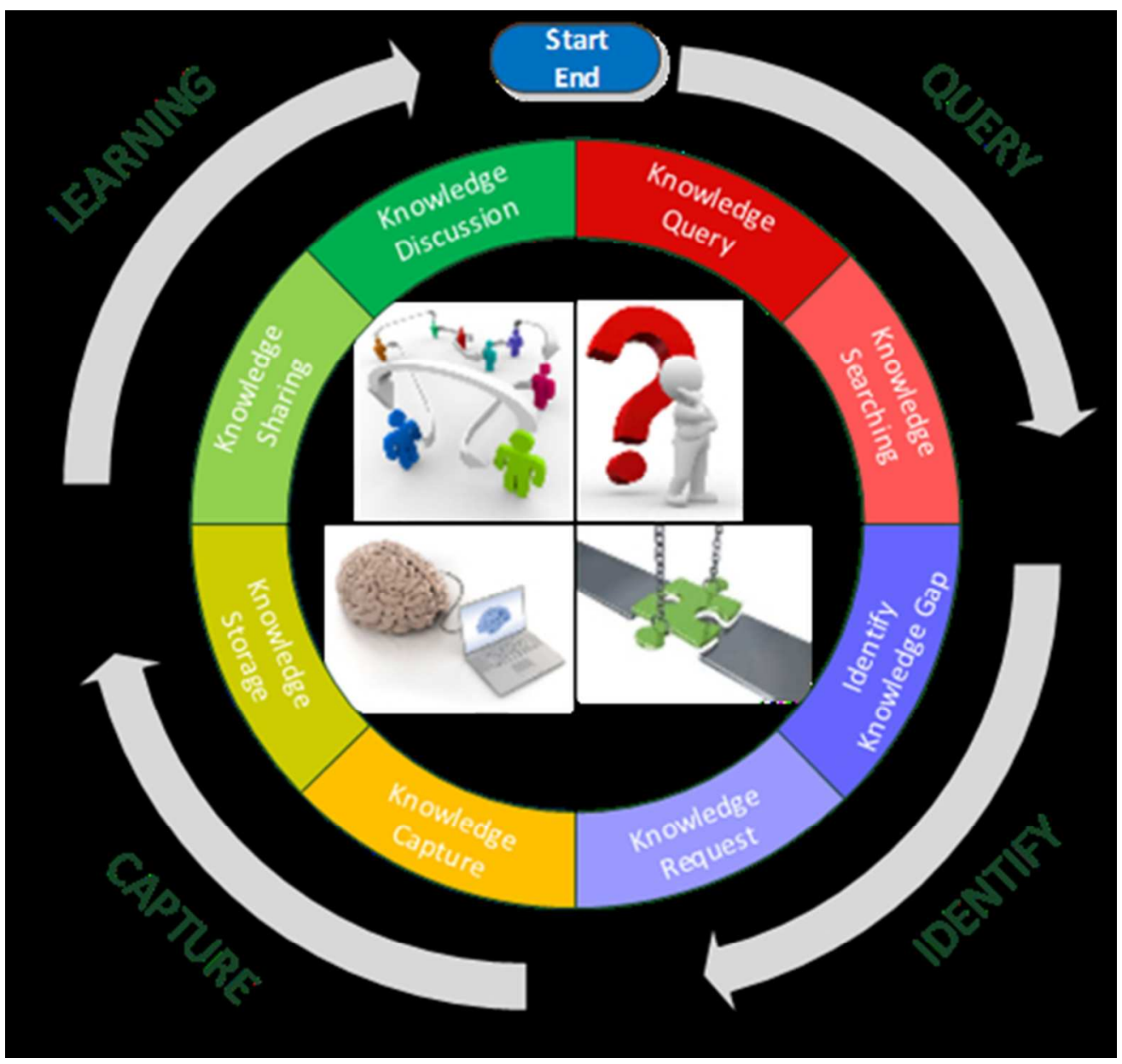

Knowledge Framework to support the PD team Figure 1 


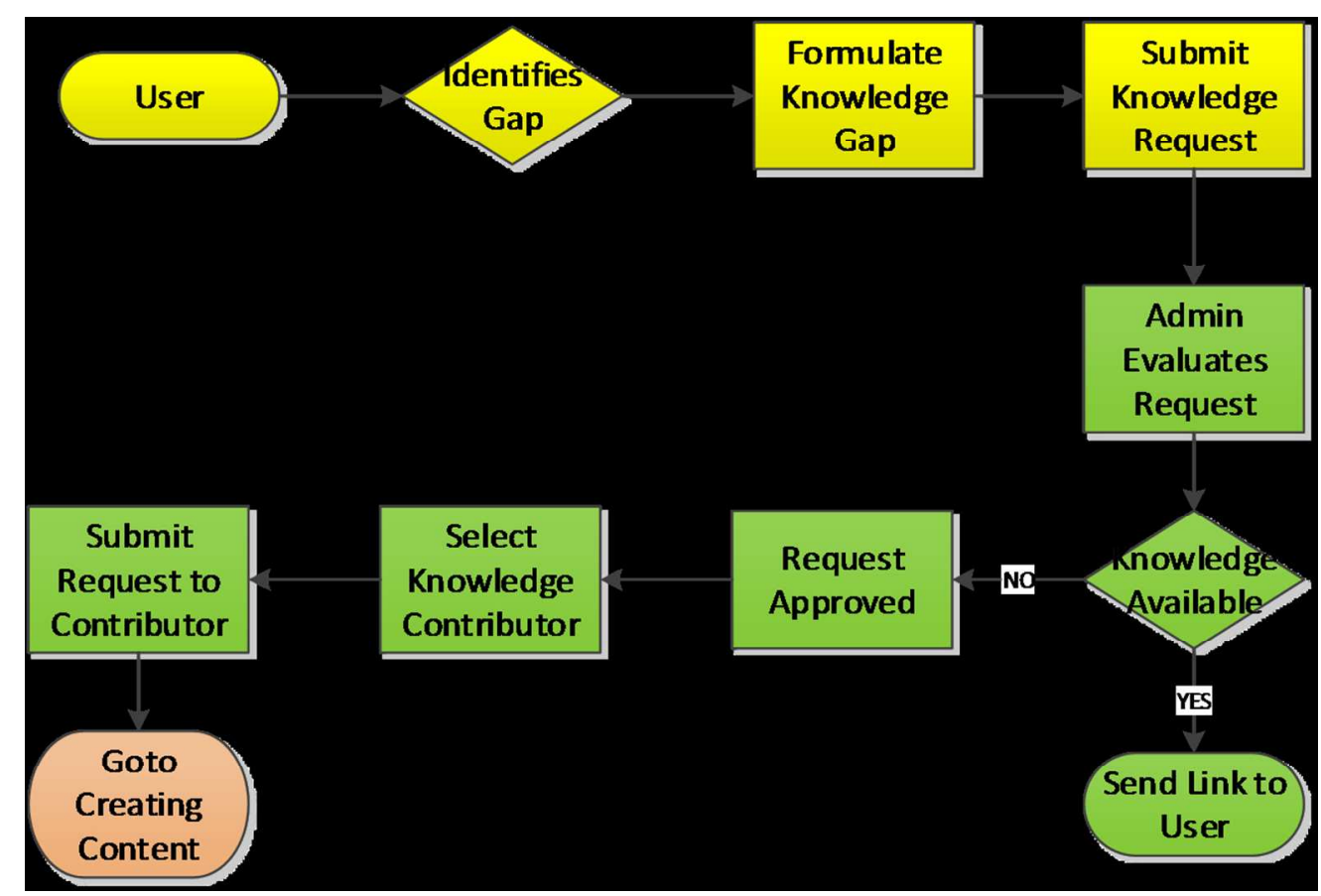

Knowledge user - Knowledge request process flow Figure 2 


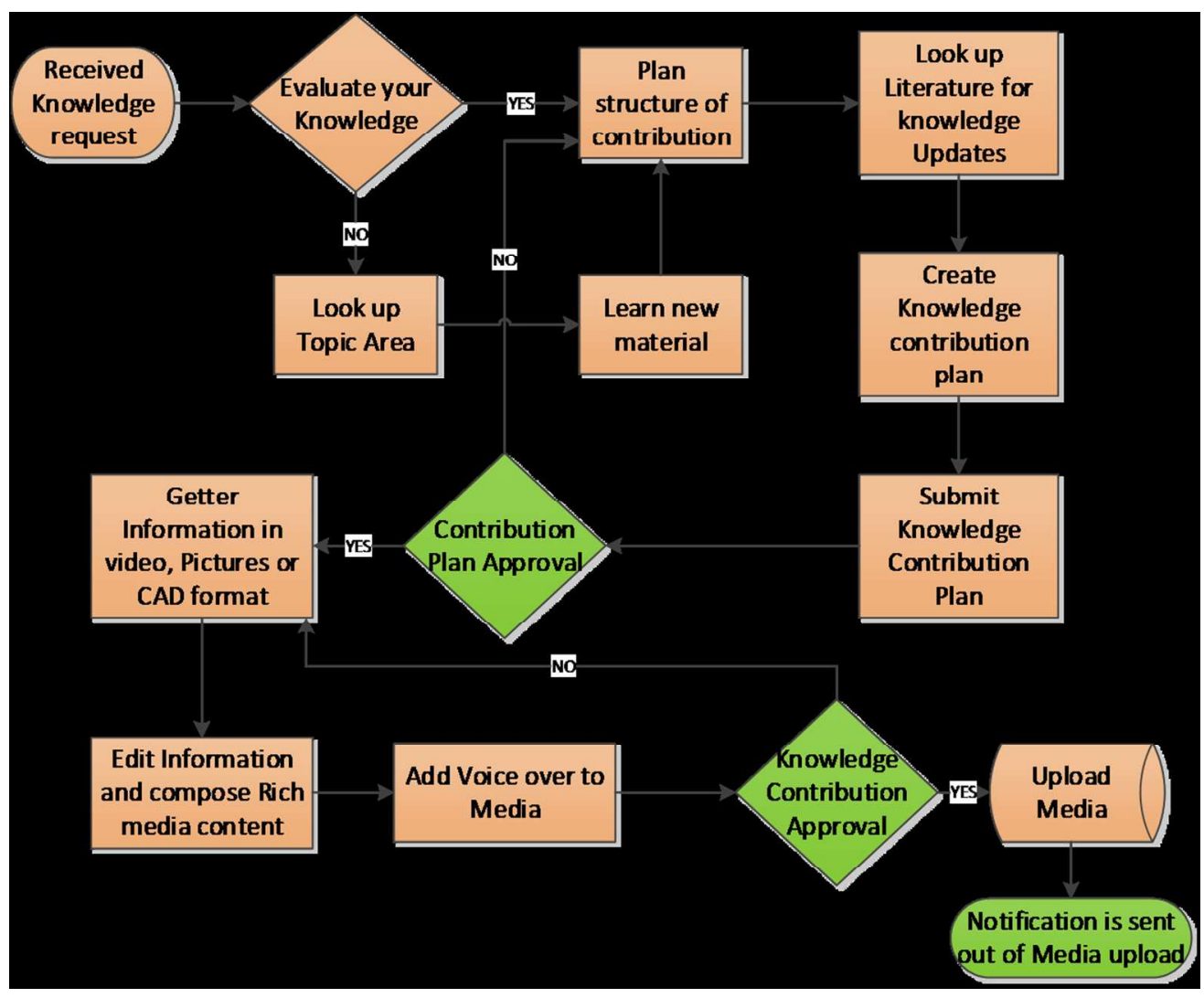

Knowledge contributor - Knowledge capture process flow Figure 3 


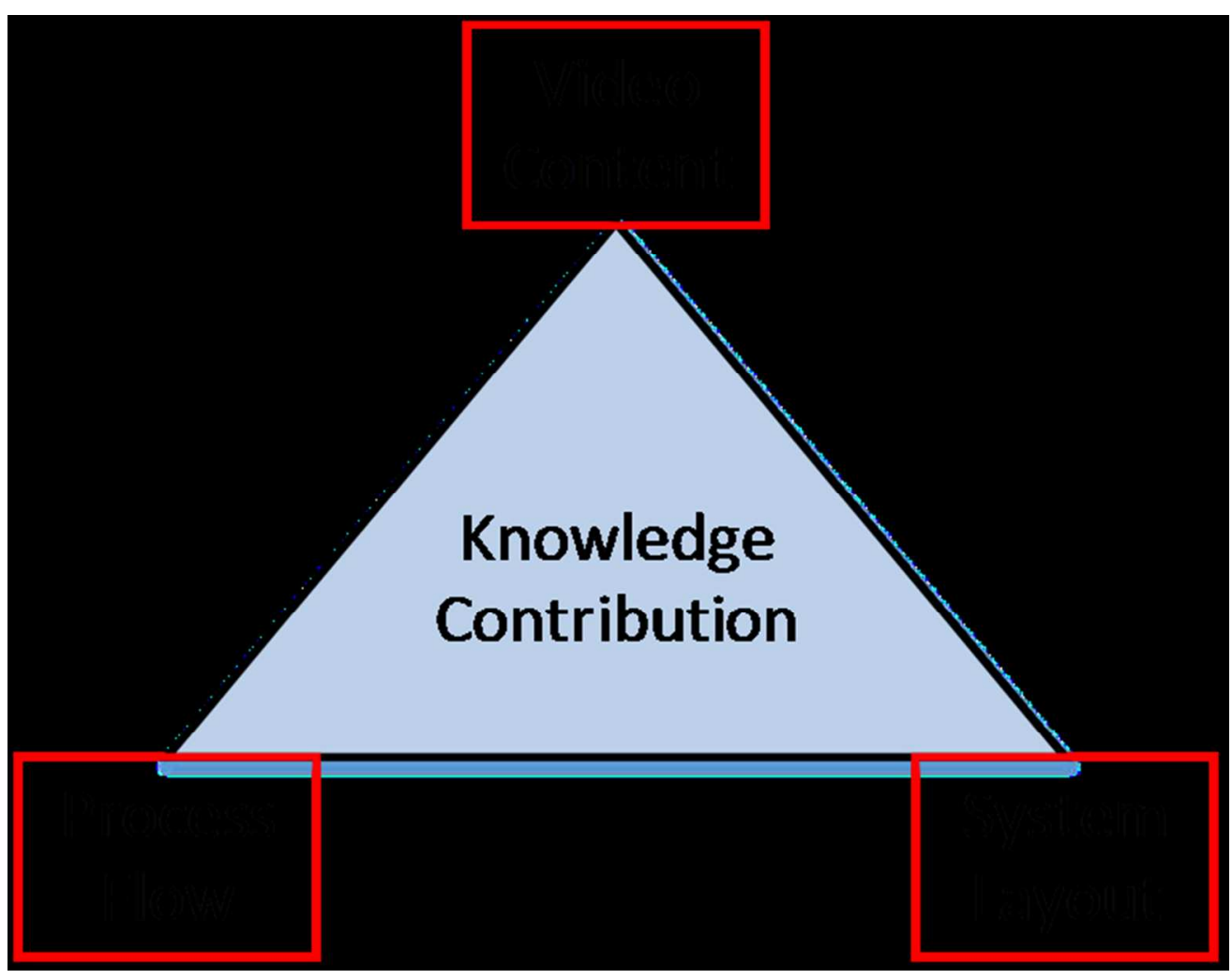

Knowledge contribution main components

Figure 4 


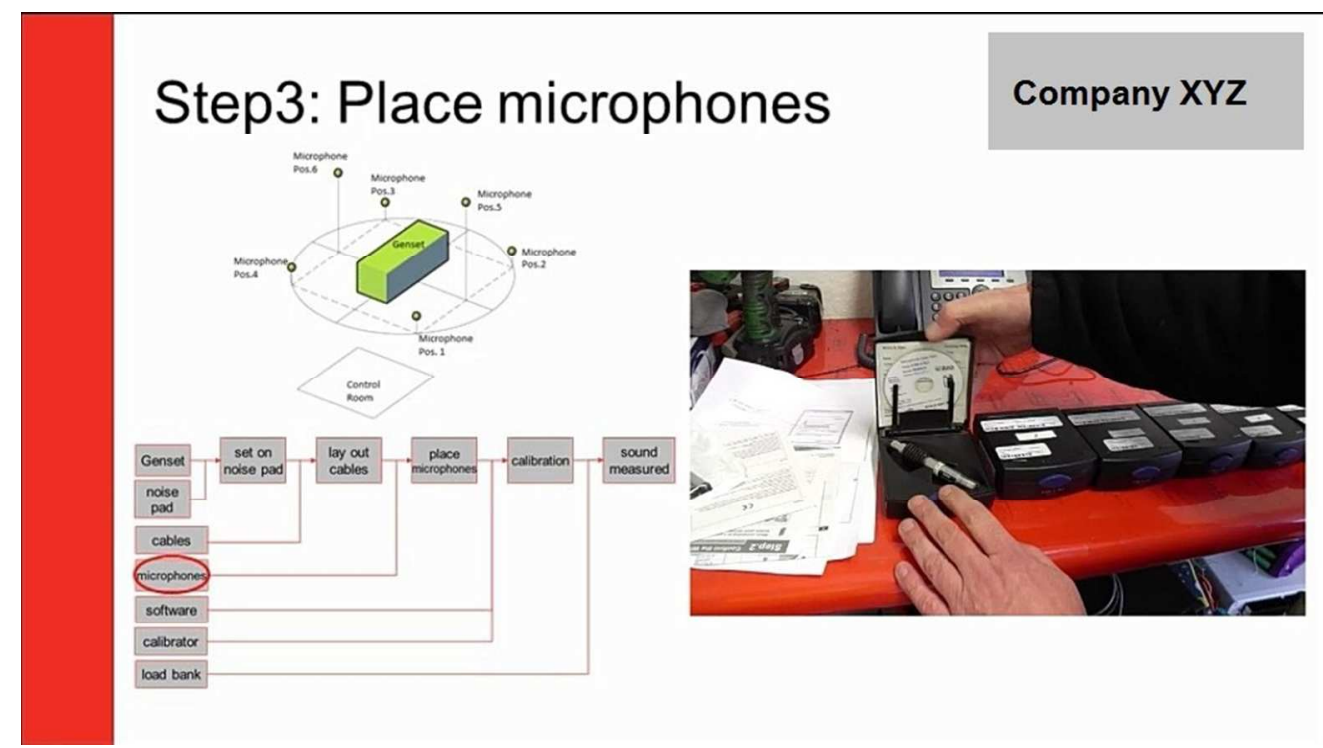

Knowledge contribution template - complete example Figure 5 $268 \times 151 \mathrm{~mm}(96 \times 96 \mathrm{DPI})$ 
Difficulty to Create Knowledge Contribution Figure 7

$69 \times 42 \mathrm{~mm}(220 \times 220$ DPI $)$ 
Difficulty to Capture Knowledge Media Figure 8

$69 \times 42 \mathrm{~mm}(220 \times 220 \mathrm{DPI})$

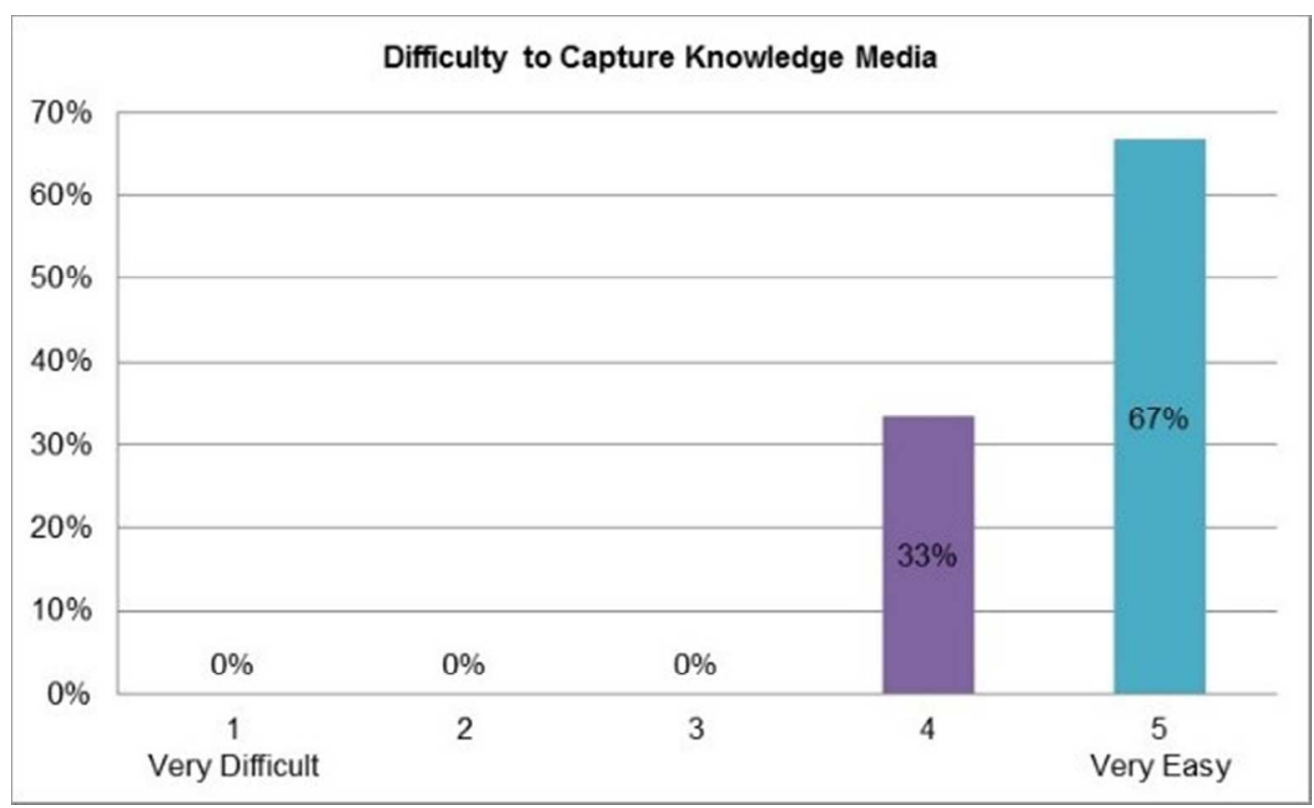


